\title{
Effects and safety of triamcinolone acetonide- controlled common therapy in keloid treatment: a Bayesian network meta-analysis
}

This article was published in the following Dove Press journal:

Therapeutics and Clinical Risk Management

\author{
Zhenyu Zhang' \\ Lihui Cheng ${ }^{2}$ \\ Ru Wang' \\ Ying Cen' \\ Zhengyong $\mathrm{Li}^{\prime}$
}

'Department of Aesthetic Plastic and Burn Surgery, West China Hospital,

Sichuan University, Chengdu, People's

Republic of China; ${ }^{2}$ Department

of Pancreatic Surgery, West China

Hospital, Sichuan University, Chengdu,

People's Republic of China
Correspondence: Zhengyong Li Department of Aesthetic Plastic and Burn Surgery, West China Hospital, Sichuan University, No 37 Guo Xue Xiang, Wuhou, 61004I Chengdu, Sichuan, People's Republic of China

Tel +86 I56 29054695

Email 15629054695m@sina.cn
Background: Triamcinolone acetonide (TAC) is used frequently in the treatment of keloid scars, but has presented controversial results. In this study, we aim to evaluate the effectiveness of TAC compared with other common therapies used in keloid treatment.

Methods: MEDLINE, Embase, Web of Science and the Cochrane Library databases were searched until January 2018. Key data were extracted from eligible randomized controlled trials. Both pairwise and network meta-analyses were conducted for synthesizing data from eligible studies.

Results: Ten randomized controlled trials were included in this meta-analysis. The relative risk of keloids associated with seven adjuvants was analyzed, including placebo, pulsed dye laser (PDL), 5-fluorouracil (5-FU), silicone, verapamil, TAC+5-FU and TAC+5-FU+PDL. Patients treated with the following adjuvants appeared to not have significantly reduced risk of keloid in relation to those treated with TAC: placebo $(\mathrm{OR}=1.86,95 \%$ CI 1.12-2.61), PDL $(\mathrm{OR}=1.32$, 95\% CI 0.53-3.30), 5-FU ( $\mathrm{OR}=1.13$, 95\% CI 0.48-2.68), silicone (OR=1.28, 95\% CI 0.59-2.78), verapamil ( $\mathrm{OR}=1.86,95 \% \mathrm{CI} 0.67-5.14), \mathrm{TAC}+5-\mathrm{FU}(\mathrm{OR}=0.77,95 \% \mathrm{CI}$ $0.38-1.58)$ and TAC+5-FU+PDL (OR=0.80, 95\% CI 0.16-4.03). The surface under the cumulative ranking curve values for each adjuvant were as follows: TAC, 59.9\%; placebo, 17.4\%; PDL, 46.3\%; 5-FU, 48.9\%; silicone, 56.2\%; verapamil, 84.7\%; TAC+5-FU, $68.5 \%$ and TAC+5-FU+PDL, $18.1 \%$.

Conclusion: There were no differences between the efficacy of TAC and other common therapies in keloid treatment. TAC also acts as an effective alternative modality in the prevention and treatment of keloids. Incorporating adjuvants particularly verapamil appeared to be significantly associated with a decreased risk of keloids.

Keywords: keloid, triamcinolone acetonide, randomized controlled trial, network meta-analysis

\section{Introduction}

Keloids are caused by the uncontrolled deposition of collagen and glycosaminoglycans around the wounds on the dermal dermis of the skin. ${ }^{1}$ During wound healing, the balance between the anabolic and catabolic effects of collagen is destroyed due to various causes forming a pathological scar. ${ }^{2,3}$ Keloids are caused by the proliferation of fibrogenic cells and the formation of large extracellular matrix, and their development is characterized by excessive collagen synthesis and deposition. ${ }^{4}$ Keloids can bring psychological and physical pain to patients from the aspect of appearance and body function. In severe cases, keloids even affect the self-confidence of patients leading to inferiority complex. ${ }^{4}$ Therefore, in the burn trauma, plastic surgery and dermatology department, keloid is 
the focus of high clinical attention. Although drugs and treatments are currently available for keloids, there are enormous challenges in their prevention and treatment, and there is no satisfactory universal treatment for all keloids. ${ }^{5}$

Corticosteroids are highly bioactive substances secreted by the adrenal cortex through tissue fluid and blood, which play a powerful role in physiological regulation. ${ }^{6}$ Most of them are hormones, such as glucocorticosteroids and sex hormones. They have anti-inflammatory, anti-allergy, antidrug, anti-nuclear fission and other effects, and their role is strong and lasting. ${ }^{7-9}$ Corticosteroids are most commonly used as injections in the early stage of the maturation phase, and intralesional injection of the corticosteroid triamcinolone acetonide (TAC) is one of the first-line treatment modalities for keloid treatment. ${ }^{10}$

The use of TAC in keloid treatment is commonplace. Wong et al found TAC therapy reduces the incidence of keloids among patients. ${ }^{11}$ However, the efficacy of TAC has not been compared with other common therapies efficacious in treating keloids. Therefore, to determine the efficacy and safety of TAC in keloid treatment compared to other common therapies, we conducted a network meta-analysis based on randomized controlled trials.

\section{Methods}

\section{Search strategy}

Eligible studies were systematically searched in MEDLINE, Embase, Web of Science and the Cochrane Library databases until January 2018 with keywords including "Keloid” [MeSH] OR “Acne Keloid” [MeSH] OR "Hypertrophic" [MeSH] OR "Scar" [MeSH] AND "triamcinolone acetonide" [MeSH] OR “Corticosteroids" [MeSH] OR “steroids" [MeSH] AND "Randomized Controlled Trial" [MeSH].

\section{Inclusion criteria}

The studies that met the following inclusion criteria were included in the meta-analysis: (1) the study must have included keloid patients; (2) the relationship between TAC and keloid must have been studied; and (3) the study must be a randomized controlled trial.

\section{Statistical analysis}

We conducted a network meta-analysis (Bayesian approach) which included both direct and indirect evidence in the network. Adjuvants were ranked based on the surface under the cumulative ranking curve (SUCRA) values. One adjuvant is more preferable than the other if it has a larger SUCRA

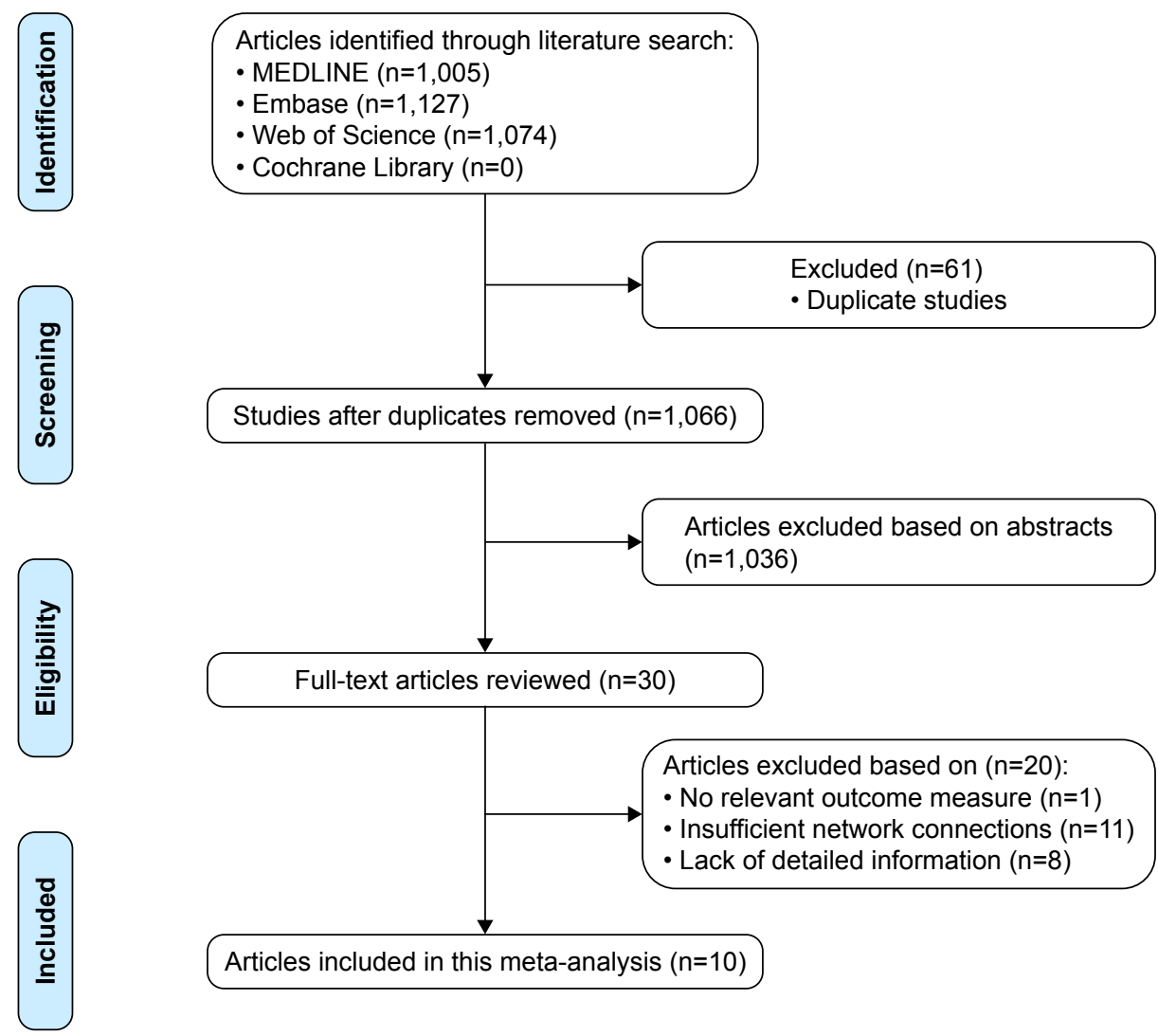

Figure I Flow diagram of the study selection process. 
value. Small study effects or publication bias were visually inspected using the funnel plots.

\section{Results}

\section{Literature search results}

A total of 1,005 studies from MEDLINE, 1,127 studies from Embase and 1,074 studies from Web of Science were selected. After removing duplicates, 1,066 studies were identified. After reviewing their titles and abstracts, 1,029 citations were excluded. The remaining 37 citations were assessed in more detail for eligibility by reading the full text. Among them, one study was excluded due to no relevant outcome measure, 11 studies were excluded due to insufficient network connections and eight studies were excluded due to lack of detailed information. Finally, 10 studies were used for the final data synthesis. ${ }^{12-21}$ The flowchart of literature search is presented in Figure 1, and the risk of bias of eight studies included in this meta-analysis is summarized in Figure 2. The characteristics of the included studies are shown in Table 1, with the pattern of evidence within the network displayed in Figure 3.

\section{Results of pairwise meta-analysis}

Table 2 displays the results produced by pairwise metaanalysis. Patients treated with the following seven adjuvants appeared to not have significantly reduced risk of keloids in relation to those treated with TAC: placebo $(\mathrm{OR}=1.86$, 95\% CI 1.12-2.61), pulsed dye laser (PDL; OR $=1.32,95 \%$ CI 0.53-3.30), 5-fluorouracil (5-FU; OR $=1.13,95 \%$ CI $0.48-2.68)$, silicone $(\mathrm{OR}=1.28,95 \%$ CI $0.59-2.78)$, verapamil (OR $=1.86,95 \%$ CI $0.67-5.14), \mathrm{TAC}+5-\mathrm{FU}(\mathrm{OR}=0.77$, 95\% CI $0.38-1.58)$ and $\mathrm{TAC}+5-\mathrm{FU}+\mathrm{PDL}(\mathrm{OR}=0.80$, 95\% CI 0.16-4.03). Patients treated with the following adjuvants appeared to have a significantly reduced risk of keloids in relation to those treated with placebo: PDL ( $\mathrm{OR}=0.34,95 \%$ CI $0.27-0.43), 5-\mathrm{FU}(\mathrm{OR}=0.37,95 \% \mathrm{CI}$ $0.26-0.52)$, silicone $(\mathrm{OR}=0.40,95 \%$ CI $0.29-0.54)$ and $\mathrm{TAC}+5$-FU (OR=0.49, 95\% CI 0.28-0.85). Moreover, there was no significant heterogeneity among studies for the abovementioned significant results $(P$-heterogeneity $>0.05$ and $I^{2}<50 \%$ ).

\section{Network meta-analysis}

Table 3 displays the results produced by network metaanalysis. Patients treated with the following seven adjuvants appeared to have a significantly reduced risk of keloids in relation to those treated with placebo: $\mathrm{TAC}(\mathrm{OR}=0.08$, 95\% CI 0.00-0.18), PDL (OR=0.30, 95\% CI 0.06-0.55),

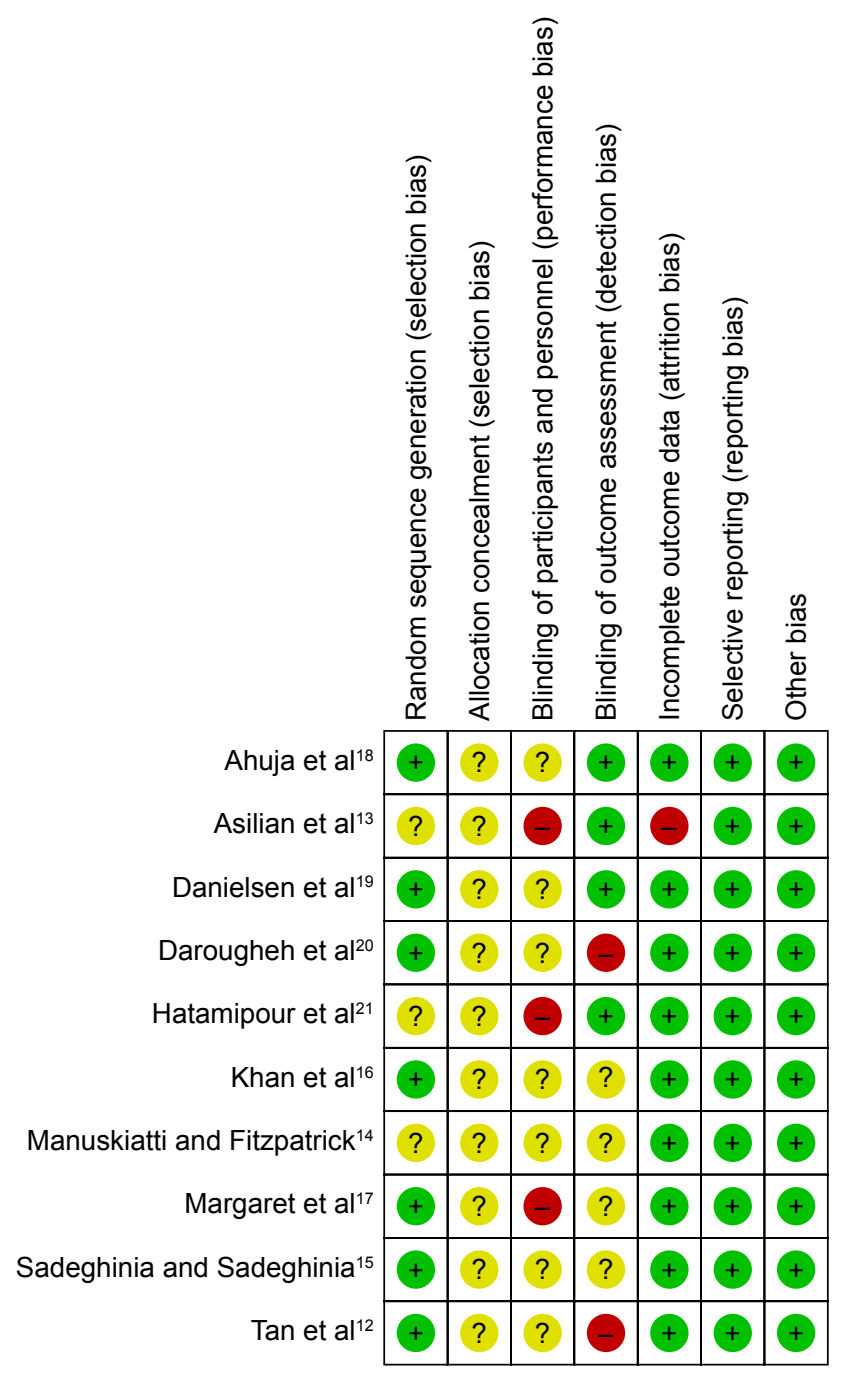

Figure 2 Risk of bias of the included randomized controlled trials (judgments about each risk-of-bias item for each included study: +, low risk; -, high risk; ?, unclear risk).

5-FU (OR $=0.32,95 \%$ CI 0.05-0.78), silicone ( $\mathrm{OR}=0.20,95 \%$ CI 0.01-0.40), verapamil ( $\mathrm{OR}=0.23,95 \%$ CI $0.01-0.46$ ), $\mathrm{TAC}+5-\mathrm{FU}(\mathrm{OR}=0.12,95 \% \mathrm{CI} 0.01-0.24)$ and $\mathrm{TAC}+5$ $\mathrm{FU}+\mathrm{PDL}(\mathrm{OR}=0.46,95 \%$ CI $0.27-0.77)$.

The corresponding SUCRA values are presented in Figure 4. The adjuvants were ranked based on SUCRA values as follows: TAC, $59.9 \%$; placebo, $17.4 \%$; PDL, 46.3\%; 5-FU, 48.9\%; silicone, $56.2 \%$; verapamil, $84.7 \%$; TAC+5-FU, $68.5 \%$; and TAC+5-FU+PDL, $18.1 \%$. Incorporating adjuvants particularly verapamil appeared to be significantly associated with a decreased risk of keloids.

\section{Publication bias}

The result of the comparison-adjusted funnel plots did not reveal any evidence of apparent asymmetry (Figure 5). No significant publication bias was observed. 


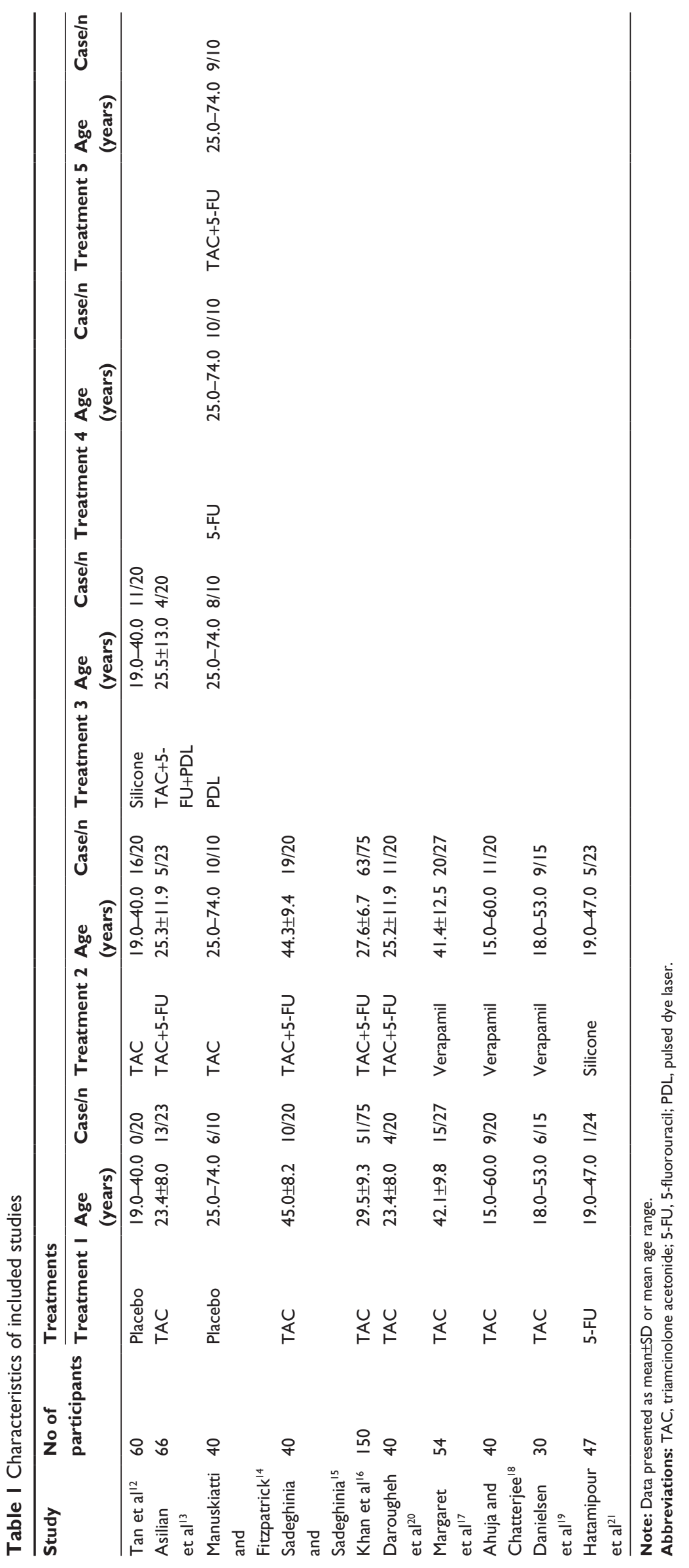




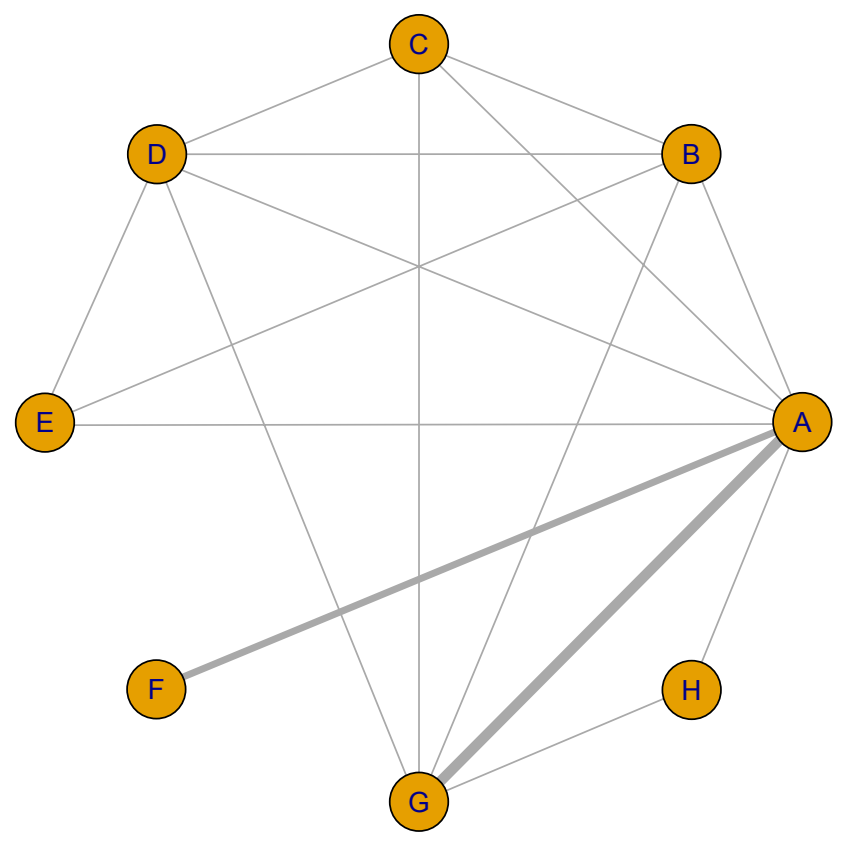

Figure 3 Network of randomized controlled trials comparing different adjuvant therapies for keloid treatment.

Notes: The thickness of the connecting lines represents the number of trials between each comparator, and the size of each node corresponds to the number of subjects who received the same pharmacological agent (sample size). (A: TAC; B: placebo: C: PDL; D: 5-FU; E: silicone; F: verapamil; G: TAC+5-FU; H: TAC+5-FU+PDL). Abbreviations: TAC, triamcinolone acetonide; PDL, pulsed dye laser; 5-FU, 5-fluorouracil.

\section{Discussion}

Objective and reliable clinical evaluation methods and prevention and control measures for scars are still a hot issue. Most of the scar treatments have achieved good results in the past 20 years, but few have been supported by prospective surveys in the control group, and some of them even lack safety data. Many new therapies have shown early effects in small-sample trials, but these effects have not been confirmed with long-term follow-up and large samples. In recent years, the understanding of wound healing and scar formation has deepened. The accumulation of a great deal of clinical experience in scar treatment, and the research and application of new preparations and new treatment methods, especially the emerging technologies, have subverted some traditional treatment concepts and thus require the establishment of safe and effective standardized scar management protocols that can be used in routine clinical practice to guide clinical treatment. ${ }^{22-24}$ The mechanism of scar formation is not fully understood, but the relevant cognitive exploration in micro and macro aspects is ongoing. The whole process of scar formation involves not only micro cells (fibroblasts, myofibroblasts, mast cells, neutrophils, etc.) but also cytokines (transforming growth factor beta, tumor necrosis factor alpha, vascular endothelial growth factor, etc.), extracellular matrix components (collagen metabolism and arrangement of arrhythmia, the change in glycosaminoglycan interactions) and organization space structure (space regulation network of repair cells formed between the three dimensions). ${ }^{25,26}$ Surgical procedures are not the best choice for keloid management as the recurrence rate is high. As alternatives to the use of corticosteroids, chemotherapeutic agents, verapamil, silicone gel tablets and cryotherapy have become important, particularly in patients with high recurrence rates after surgery. ${ }^{10}$ Khansa et al performed a meta-analysis and showed silicone gel, PDL, TAC and 5-FU had high efficacy in improving keloids. ${ }^{27}$ However, which common therapy was the most effective in improving keloids was unknown.

Table 2 Summary ORs of TAC and heterogeneity for each direct comparison

\begin{tabular}{|c|c|c|c|c|}
\hline Comparison & OR $(95 \% \mathrm{Cl})$ & P-heterogeneity & $I^{2}$ & $\tau^{2}$ \\
\hline Placebo vs TAC & $1.86(1.12-2.61)$ & 0.697 & $<0.01 \%$ & $<0.001$ \\
\hline PDL vs TAC & I.32 (0.53-3.30) & 0.286 & $20.7 \%$ & 0.112 \\
\hline 5-FU vs TAC & I.I $3(0.48-2.68)$ & 0.723 & $<0.01 \%$ & 0.317 \\
\hline Silicone vs TAC & $1.28(0.59-2.78)$ & 0.790 & $<0.01 \%$ & 0.713 \\
\hline Verapamil vs TAC & $1.86(0.67-5.14)$ & 0.211 & $29.9 \%$ & 0.541 \\
\hline TAC+5-FU vs TAC & $0.77(0.38-1.58)$ & 0.160 & $37.0 \%$ & 0.759 \\
\hline $\mathrm{TAC}+5-\mathrm{FU}+\mathrm{PDL}$ vs TAC & $0.80(0.16-4.03)$ & 0.146 & $35.4 \%$ & 0.951 \\
\hline PDL vs placebo & $0.34(0.27-0.43)$ & 0.814 & $<0.01 \%$ & $<0.001$ \\
\hline Silicone vs placebo & $0.40(0.29-0.54)$ & 0.660 & $<0.01 \%$ & $<0.001$ \\
\hline 5-FU vs placebo & $0.37(0.26-0.52)$ & 0.620 & $<0.01 \%$ & $<0.001$ \\
\hline $\mathrm{TAC}+5-\mathrm{FU}$ vs placebo & $0.49(0.28-0.85)$ & - & - & $<0.001$ \\
\hline 5-FU vs PDL & $1.45(0.42-5.00)$ & 0.303 & $17.6 \%$ & 0.951 \\
\hline $\mathrm{TAC}+5-\mathrm{FU}$ vs PDL & $1.25(0.42-3.67)$ & - & - & 0.899 \\
\hline Silicone vs 5-FU & $0.86(0.30-2.4 \mathrm{I})$ & - & - & 0.691 \\
\hline $\mathrm{TAC}+5-\mathrm{FU}$ vs $5-\mathrm{FU}$ & $0.60(0.24-1.49)$ & - & - & 0.644 \\
\hline $\mathrm{TAC}+5-\mathrm{FU}+\mathrm{PDL}$ vs TAC+5-FU & $1.09(0.39-2.97)$ & - & - & 0.926 \\
\hline
\end{tabular}

Notes: Bold values indicate $P<0.05$. “- “ indicates data not available.

Abbreviations: TAC, triamcinolone acetonide; PDL, pulsed dye laser; 5-FU, 5-fluorouracil. 


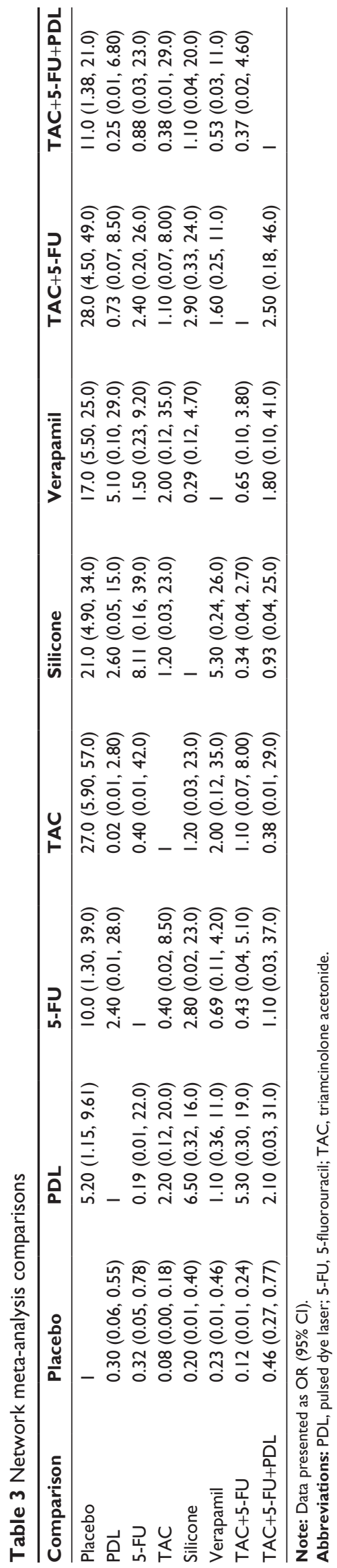

This network meta-analysis attempted to evaluate the effectiveness of TAC compared with other common therapies used in keloid treatment. Our analysis suggests that verapamil is potentially more preferable than other adjuvants. Verapamil has been used as a coronary dilator since 1962. In the recent years, it has been used for the treatment of hypertension, angina, arrhythmia, cerebrovascular disease, finger vasospasm, abdominal pain, esophageal delaying, migraine and pulmonary hypertension and to prevent preterm birth. In 1997, Dong et al first reported the treatment of keloids with calcium channel blocker verapamil, which immediately attracted the attention of clinicians. ${ }^{28}$ After 1997, attempts have been made to identify and treat keloid patients with verapamil. Verapamil has been shown to increase procollagenase synthesis in normal cultured fibroblasts. It also leads to the depolymerization of actin, cell conformation change and cell apoptosis, and ultimately leads to the reduction of fibrous tissue formation. ${ }^{29}$ Boggio et al confirmed $50 \mu \mathrm{M}$ verapamil was effective in wound healing, and it can also avoid the development of keloids and hypertrophic scars after plastic surgery. ${ }^{30}$

As suggested by the SUCRA ranking scheme, TAC+5-FU was ranked behind verapamil. 5-FU is an anti-pyrimidine drug. It has to be enzymatically converted to 5 -fluorodeoxyuridine nucleotides and exhibits antitumor activity. ${ }^{31,32}$ The action of this enzyme may also transfer one carbon unit of leucovorin to deoxyuridine nucleotide monophosphate for the synthesis of thymidine monoacid. At the same time, it also shows some inhibitory effect on the synthesis of RNA. ${ }^{31}$ Shin et al found 5-FU was more effective than TAC in keloid treatment after surgical excision. ${ }^{33}$

This meta-analysis also has some limitations. The test result of the corticosteroid therapy showed statistical heterogeneity was limited in randomized controlled trials, and limited evidence of a dose-dependent association between corticosteroids therapy and keloids, which provides limited confidence in the findings. Second, there is no record of keloid patients treated in a standardized manner, which leads to the difference between the trials; therefore, these results should be interpreted with caution. Third, study durations were short in these randomized controlled trials, and patients included in these trials may be different from real life. Fourth, these findings may not be generalizable to a specific group of patients because randomized controlled trials tend to exclude participants.

Our findings underscore the notion that any common therapy compared with TAC did not reduce keloid risk. Verapamil is potentially the most preferable adjuvant in keloid 


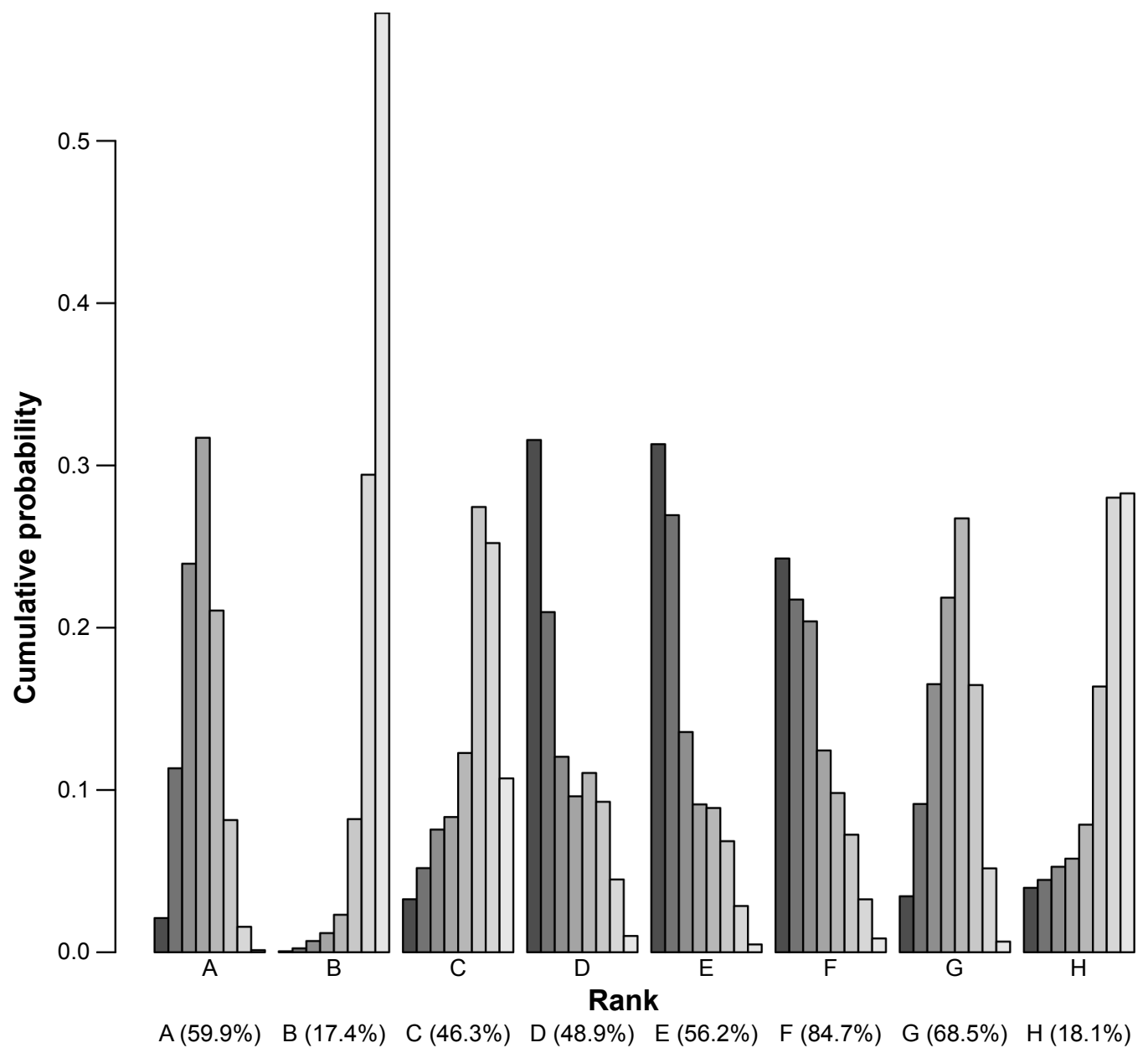

Figure 4 SUCRA values, expressed as percentages, ranking the therapeutic effects and safety of treatments for keloids.

Notes: For efficacy and safety assessment, the pharmacological agent with the highest SUCRA value would be the most efficacious and safe treatment (A: TAC; B: placebo; C: PDL; D: 5-FU; E: silicone; F: verapamil; G: TAC+5-FU; H: TAC+5-FU+PDL).

Abbreviations: SUCRA, surface under the cumulative ranking curve; TAC, triamcinolone acetonide; PDL, pulsed dye laser; 5-FU, 5-fluorouracil.

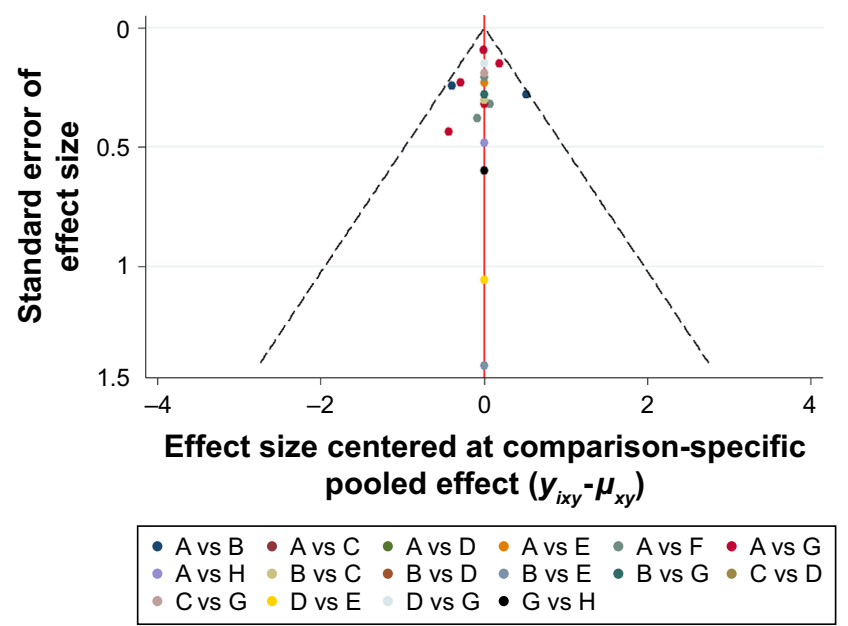

Figure 5 Comparison-adjusted funnel plot for the network meta-analysis.

Notes: The red line suggests the null hypothesis that the study-specific effect sizes do not differ from the respective comparison-specific pooled effect estimates. Different colors represent different comparisons (A: TAC; B: placebo; C: PDL; D: 5-FU; E: silicone; F: verapamil; G: TAC+5-FU; H: TAC+5-FU+PDL).

Abbreviations: TAC, triamcinolone acetonide; PDL, pulsed dye laser; 5-FU, 5-fluorouracil. treatment. In the future, large-scale trials must be performed to validate the risk identified in the current meta-analysis.

\section{Acknowledgments}

This study was supported by the Sichuan Science and Technology Department Applied Basic Research Project (grant no. 2017JY0335) and (grant no.2017JY0250).

\section{Disclosure}

The authors report no conflicts of interest in this work.

\section{References}

1. Younai S, Nichter LS, Wellisz T, Reinisch J, Nimni M, Tuan T. Modulation of collagen synthesis by transforming growth factor-beta in keloid and hypertrophic scar fibroblasts. Ann Plast Surg. 1994;33(2): $148-151$.

2. Rekha A. Keloids - a frustrating hurdle in wound healing. Int Wound $J$. 2004;1(2):145-148.

3. Diegelmann RF, Evans MC. Wound healing: an overview of acute, fibrotic and delayed healing. Front Biosci. 2004;9:283-289. 
4. Ogawa R. Mechanobiology of scarring. Wound Repair Regen. 2011; 19Suppl 1:s2-s9.

5. Majewski S, Bosch FX, Dillner J, et al. The impact of a quadrivalent human papillomavirus (types $6,11,16,18$ ) virus-like particle vaccine in European women aged 16 to 24. J Eur Acad Dermatol Venereol. 2009; 23(10):1147-1155.

6. Datey KK, Pandya VN. Corticosteroids. Med Digest. 1961;29:241-254.

7. Fernandez-Serrano $\mathrm{S}$, Dorca J, Coromines M, Carratalà J, Gudiol F, Manresa F. Molecular inflammatory responses measured in blood of patients with severe community-acquired pneumonia. Clin Diagn Lab Immunol. 2003;10(5):813-820.

8. Garcia-Vidal C, Calbo E, Pascual V, Ferrer C, Quintana S, Garau J. Effects of systemic steroids in patients with severe community-acquired pneumonia. Eur Respir J. 2007;30(5):951-956.

9. Antunes G, Evans SA, Lordan JL, Frew AJ. Systemic cytokine levels in community-acquired pneumonia and their association with disease severity. Eur Respir J. 2002;20(4):990-995.

10. Mustoe TA, Cooter RD, Gold MH, et al; International Advisory Panel on Scar Management. International clinical recommendations on scar management. Plast Reconstr Surg. 2002;110(2):560-571.

11. Wong TS, Li JZ, Chen S, Chan JY, Gao W. The efficacy of triamcinolone acetonide in keloid treatment: a systematic review and meta-analysis. Front Med. 2016;3:71.

12. Tan E, Chua S, Lim J. Topical silicone gel sheet versus intralesional injections of triamcinolone acetonide in the treatment of keloids - a patient-controlled comparative clinical trial. J Dermatol Treat. 1999; 10(4):251-254.

13. Asilian A, Darougheh A, Shariati F. New combination of triamcinolone, 5-fluorouracil, and pulsed-dye laser for treatment of keloid and hypertrophic scars. Dermatol Surg. 2006;32(7):907-915.

14. Manuskiatti W, Fitzpatrick RE. Treatment response of keloidal and hypertrophic sternotomy scars: comparison among intralesional corticosteroid, 5-fluorouracil, and 585-nm flashlamp-pumped pulseddye laser treatments. Arch Dermatol. 2002;138(9):1149-1155.

15. Sadeghinia A, Sadeghinia S. Comparison of the efficacy of intralesional triamcinolone acetonide and 5-fluorouracil tattooing for the treatment of keloids. Dermatol Surg. 2012;38(1):104-109.

16. Khan MA, Bashir MM, Khan FA. Intralesional triamcinolone alone and in combination with 5-fluorouracil for the treatment of keloid and hypertrophic scars. J Pak Med Assoc. 2014;64(9):1003-1007.

17. Margaret SFX, Ernest K, Dhanraj P. Comparison of intralesional verapamil with intralesional triamcinolone in the treatment of hypertrophic scars and keloids. Indian J Dermatol Venereol Leprol. 2008;74(4): 343-348.

18. Ahuja RB, Chatterjee P. Comparative efficacy of intralesional verapamil hydrochloride and triamcinolone acetonide in hypertrophic scars and keloids. Burns. 2014;40(4):583-588.

19. Danielsen PL, Rea SM, Wood FM, et al. Verapamil is less effective than triamcinolone for prevention of keloid scar recurrence after excision in a randomized controlled trial. Acta Derm Venereol. 2016;96(6): 774-778.
20. Darougheh A, Asilian A, Shariati F. Intralesional triamcinolone alone or in combination with 5-fluorouracil for the treatment of keloid and hypertrophic scars. Clin Exp Dermatol. 2009;34(2):219-223.

21. Hatamipour E, Mehrabi S, Hatamipour M, Ghafarian Shirazi HR. Effects of combined intralesional 5-fluorouracil and topical silicone in prevention of keloids: a double blind randomized clinical trial study. Acta Med Iran. 2011;49(3):127-130.

22. Huang C, Murphy GF, Akaishi S, Ogawa R. Keloids and hypertrophic scars: update and future directions. Plast Reconstr Surg Glob Open. 2013;1(4):e25.

23. Ogawa R. The most current algorithms for the treatment and prevention of hypertrophic scars and keloids. Plast Reconstr Surg. 2010; 125(2):557-568.

24. Klinger M, Marazzi M, Vigo D, Torre M. Fat injection for cases of severe burn outcomes: a new perspective of scar remodeling and reduction. Aesthetic Plast Surg. 2008;32(3):465-459.

25. Wang X, Smith P, Pu LL, Kim YJ, Ko F, Robson MC. Exogenous transforming growth factor beta(2) modulates collagen I and collagen III synthesis in proliferative scar xenografts in nude rats. J Surg Res. 1999;87(2):194-200.

26. Oriente A, Fedarko NS, Pacocha SE, Huang SK, Lichtenstein LM, Essayan DM. Interleukin-13 modulates collagen homeostasis in human skin and keloid fibroblasts. J Pharmacol Exp Ther. 2000; 292(3):988-994.

27. Khansa I, Harrison B, Janis JE. Evidence-based scar management: how to improve results with technique and technology. Plast Reconstr Surg. 2016;138(3 Suppl):165S-178S.

28. Dong H, Earle ML, Jiang Y, Loutzenhiser KA, Triggle CR. Cardiovascular effects of CPU-23, a novel L-type calcium channel blocker with a unique molecular structure. Br J Pharmacol. 1997;122(7): 1271-1278.

29. Berman B, Maderal A, Raphael B. Keloids and hypertrophic scars: pathophysiology, classification, and treatment. Dermatol Surg. 2017; 43 Suppl 1:S3-S18.

30. Boggio RF, Boggio LF, Galvao BL, Machado-Santelli GM. Topical verapamil as a scar modulator. Aesthetic Plast Surg. 2014;38(5): 968-975.

31. de Waard JW, de Man BM, Wobbes T, van der Linden CJ, Hendriks T. Inhibition of fibroblast collagen synthesis and proliferation by levamisole and 5-fluorouracil. Eur J Cancer. 1998;34(1):162-167.

32. Al-Attar A, Mess S, Thomassen JM, Kauffman CL, Davison SP. Keloid pathogenesis and treatment. Plast Reconstr Surg. 2006;117(1): 286-300.

33. Shin JY, Kim JS. Could 5-fluorouracil or triamcinolone be an effective treatment option for keloid after surgical excision? A meta-analysis. J Oral Maxillofac Surg. 2016;74(5):1055-1060.
Therapeutics and Clinical Risk Management

\section{Publish your work in this journal}

Therapeutics and Clinical Risk Management is an international, peerreviewed journal of clinical therapeutics and risk management, focusing on concise rapid reporting of clinical studies in all therapeutic areas, outcomes, safety, and programs for the effective, safe, and sustained use of medicines. This journal is indexed on PubMed Central, CAS,

\section{Dovepress}

EMBase, Scopus and the Elsevier Bibliographic databases. The manuscript management system is completely online and includes a very quick and fair peer-review system, which is all easy to use. Visit $\mathrm{http}: / / \mathrm{www}$.dovepress.com/testimonials.php to read real quotes from published authors. 